\title{
Rasgos de personalidad en judokas senior y diferencias con población general
}

\author{
Personality traits in senior judokas and \\ differences with the general population
}

\section{Traços de personalidade nos judocas sênior e diferenças com a população geral}

\author{
Gonzalo Ezequiel Torres de Olazábal ${ }^{1}$ \\ https://orcid.org/0000-0002-4482-8745
}

Cristian Manfre https://orcid.org/0000-0002-1835-8321 Roberto Oscar Sánchez \&
https://orcid.org/0000-0002-5251-4973 Macarena Verónica Del Valle
https://orcid.org/0000-0003-3549-7224

1 Torres de Olazábal, Gonzalo Ezequiel. Facultad de Psicología de la Universidad Nacional de Mar del Plata. Licenciado en Psicología por la UNMDP. Desarrolló su Trabajo de Investigación Final en el área de la evaluación de los rasgos de personalidad de deportistas de judo en el marco de un proyecto de investigación del Instituto de Investigación en Psicología Básica, Aplicada y Tecnología. Correo electrónico.: gonzalotdo@hotmail.com

- Manfre, Cristian. Facultad de Psicología de la Universidad Nacional de Mar del Plata. Licenciado en Psicología por la UNMDP. Desarrolló su Trabajo de Investigación Final en el área de la evaluación de los rasgos de personalidad de deportistas de judo en el marco de un proyecto de investigación del Instituto de Investigación en Psicología Básica, Aplicada y Tecnología. Correo electrónico.: cristianmanfre@hotmail.com Sánchez, Roberto Oscar. Instituto de Investigación en Psicología Básica, Aplicada y Tecnología (IPSIBAT - UNMDP - CONICET). Lic. en Psicología y Esp. en Docencia Universitaria. Posgrado de Formación y entrenamiento en Psicoterapia - Modelo Cognitivo Social, Fundación Aiglé. Docente Psicología Cognitiva e Introducción a la Investigación Psicológica (Fac. de Psicología, UNMdP). Director del Proyecto de Investigación "Modelos dimensionales de personalidad patológica. Aspectos conceptuales y metodológicos (Fac. de Psicología, UNMdP). Autor de "Correr: físico más mente. Factores psicológicos relacionados al correr" (EUDEM, 2019). Correo electrónico.: roserto@gmail.com / rosanche@mdp.edu.ar

- Del Valle, Macarena Verónica. Instituto de Investigación en Psicología Básica, Aplicada y Tecnología (IPSIBAT - UNMDP - CONICET). Licenciada en Psicología por la Universidad Nacional de Mar del Plata (UNMDP). Especialización en Psicoterapia Cognitiva en la Fundación Aiglé, Argentina. Master en Investigación en Educación en la Universidad de Valladolid, España. Actualmente, estudiante del Doctorado en Psicología de la UNMDP y Becaria Doctoral del CONICET. Docente en la cátedra de Teorías del Aprendizaje de la UNMDP e integrante de proyectos de investigación del IPSIBAT. Correo electrónico.: mdelvalle1989@gmail.com 
https://doi.org/10.53287/wtvc8143ci23f

Fecha de ingreso: 26 de noviembre de 2020

Fecha de aprobación: 4 de mayo de 2021

Conflictos de interés: Los autores declaran no tener conflictos de interés

\title{
RESUMEN
}

El análisis de los aspectos psicológicos de la práctica deportiva resulta de interés para el desarrollo de intervenciones orientadas a alcanzar el máximo potencial de los deportistas. Este estudio tuvo por objetivo analizar los rasgos de personalidad de judokas senior en función de (a) sexo, (b) edad, (c) graduación alcanzada, (d) máximo nivel de competición alcanzado, (e) años de experiencia, (f) categoría de peso, y (g) explorar las diferencias con los rasgos de personalidad de población general. Participaron 116 judokas argentinos (86 hombres y 30 mujeres). Se aplicó el Listado de Adjetivos para Evaluar la Personalidad. Los resultados muestran diferencias entre pequeñas y moderadas entre los deportistas en función del sexo, la edad, la graduación de judo, el nivel de competición y los años de experiencia deportiva. No se hallaron diferencias entre las categorías de peso. También se observaron diferencias con la personalidad de la población normotípica: mujeres judokas reportaron mayor Apertura y hombres judokas, mayor Amabilidad, Responsabilidad y Estabilidad emocional. Los resultados representan un aporte al conocimiento del perfil psicológico de los judokas y los rasgos presentes en deportistas de alta competición.

\section{PALABRAS CLAVE}

Deportes; Judo; Modelo Cinco Grandes Factores; Personalidad; Sexo.

\begin{abstract}
The analysis of the psychological aspects of different sports is relevant for the development of interventions aimed at reaching the maximum potential of athletes. The aim of this study was to analyze the personality traits of senior judokas according to (a) sex, (b) age, (c) graduation, (d) competition level attained, (e) years of experience, (f) weight category, and $(\mathrm{g})$ to explore the differences with the personality traits of the general population. A total of 116 Argentinean judokas (86 men and 30 women) participated in the study. Personality traits were assessed through the Adjectives Check List. The results show small to moderate differences between the judokas according to sex, age, graduation, competition level, and years of sport experience. No differences were found according to weight category. Differences in personality were also detected between the judokas and the general population: female judokas reported higher levels of Openness to experience, and male judokas reported higher levels of Agreeableness, Conscientiousness, and Emotional stability. The results represent a contribution about the psychological profile of judokas and the personality traits of high competition athletes.
\end{abstract}

\section{KEY WORDS}

Big Five Factor; Judo; Personality; Sex; Sports.

\section{RESUMO}

A análise dos aspectos psicológicos da prática desportiva é de interesse para o 
desenvolvimento de intervenções destinadas a alcançar o máximo potencial dos atletas. O objectivo deste estudo foi analisar os traços de personalidade dos judocas sênior de acordo com (a) sexo, (b) idade, (c) graduação, (d) nível de competição atingido, (e) anos de experiência, (f) categoria de peso, e (g) explorar as diferenças com os traços de personalidade da população em geral. Participaram no estudo 116 judocas argentinos (86 homens, 30 mulheres) e foram avaliados através da Lista de Adjetivos para Avaliar a Personalidade. Os resultados mostram diferenças pequenas a moderadas entre os judocas de acordo com o sexo, idade, graduação, nível de competição e anos de experiência desportiva. Não foram encontradas diferenças de acordo com a categoria de peso. Foram também encontradas diferenças de personalidade entre os judocas e a população em geral: as judocas femininas reportaram mais Abertura, e os judocas masculinos reportaram mais, Conscienciosidade e Estabilidade Emocional. Os resultados representam uma contribuição para o conhecimento do perfil psicológico dos judocas e dos traços presentes nos atletas de alta competição.

\section{PALAVRAS-CHAVE}

Desporto; Judo; Modelo Cinco Grandes Fatores; Personalidade; Sexo.

\section{Introducción}

La personalidad es considerada como una de las variables más determinantes para explicar y predecir el comportamiento humano (Jones, Miller, y Lynam, 2011; Kokko, Tolvanen, y Pulkkinen, 2013; Steca, Baretta, Greco, D’Addario, y Monzani, 2018), por lo que distintos estudios han indagado acerca de la relación entre la personalidad y la práctica deportiva (Alexandru y Răşcanu, 2013; Allen, Greenlees, y Jones, 2013; Catalá y Peñacoba, 2020; De Vries, 2020; Gee, Marshall, y King, 2010; Trninic, Barančić, y Nazor, 2008; Weinberg y Gould, 2010; Zhang et al., 2019). En particular, se ha estudiado la personalidad de los deportistas exitosos (e.g., Waleriańczyk y Stolarski, 2021), las diferencias entre la personalidad de los deportistas y la población general (e.g., García-Naveira y Ruiz-Barquín, 2016; Malinauskas, Dumciene, Mamkus, y Venckunas, 2016; Mladenović, Lazarević, Trunić, Bogavac, y Živković, 2017), se han comparado las características de personalidad entre deportistas de distintas disciplinas (e.g., Bara Filho, Scipião Ribeiro, y Guillen García, 2004; García-Naveira, Locatelli Dalimier, Ruiz-Barquín, y Gon- zález, 2016), entre deportes de grupo e individuales (e.g., González, Garcés de los Fayos, López-Mora, y Zapata, 2016; González Hernández y Valadez Jiménez, 2016; Ilyasi y Salehian, 2011; Nia y Besharat, 2010), y se ha indagado cuáles son los aspectos de la personalidad que predicen la participación y la calidad deportiva (e.g., García-Naveira y Díaz-Morales, 2010; Parmigiani et al., 2009; Ruiz-Barquín y García-Naveira, 2013; Steca et al., 2018), entre otros aspectos.

Respecto a la personalidad, el Modelo de los Cinco Grandes Factores [MCF] propuesto por Costa y McCrae (Costa y McCrae, 1985; McCrae, 2017; McCrae et al., 2000; McCrae y Costa, 1990, 2021) sugiere la existencia de cinco dimensiones bipolares internas, estables y endógenas denominadas rasgos, que serían la base de la personalidad: Amabilidad / Antagonismo, Responsabilidad / Negligencia, Extraversión / Introversión, Neuroticismo / Estabilidad emocional, y Apertura a la experiencia / Convencionalismo. Las personas diferirían en la presencia más o menos marcada de estos rasgos, dando lugar a las diferencias interindividuales en la conducta (McCrae, 2017; O’Hern, Stephens, Young, 
y Koppel, 2020). Además, los estudios sugieren que tanto el sexo como la edad poseen un efecto sobre la personalidad. Así, las mujeres tienden a presentar mayores niveles de inestabilidad emocional, empatía y apertura a los sentimientos (i.e., Neuroticismo y Amabilidad) y los hombres tienden a presentar mayores niveles de asertividad y apertura a nuevas ideas (i.e., Antagonismo y Apertura a la experiencia) (Barra Almagiá, Soto Quevedo, y Schmidt Díaz, 2019; Costa, Terracciano, y McCrae, 2001; De Miguel Negredo, 2005; Langvik, Hjemdal, y Nordahl, 2016; Manga, Ramos, y Morán, 2004; Weisberg et al., 2011). Sin embargo, las diferencias en el rasgo Neuroticismo suelen ser las más pronunciadas entre los sexos y resultan consistentes a través de las culturas (Caprara et al., 1993; Church, 2016; Costa et al., 2001; De Miguel Negredo, 2005, Schmitt, Realo, Voracek, y Allik, 2008; Schmitt et al., 2017), mientras que la evidencia sobre las diferencias de sexo en el resto de los rasgos resulta más contradictoria (Schmitt et al., 2017). Por ejemplo, Sánchez y Ledesma (2013) encontraron en población general argentina que las mujeres reportan mayores niveles de Amabilidad, Extraversión y Neuroticismo, mientras que los hombres reportan mayores niveles de Apertura a la Experiencia. Sin embargo, esas diferencias fueron pronunciadas sólo en el caso de Neuroticismo, y resultaron pequeñas para los demás rasgos. Acerca de la edad, aunque la personalidad tiende a mantenerse estable con el paso de los años, existirían procesos de maduración intrínseca en los rasgos de personalidad (McCrae et al., 2000). Las personas más jóvenes tendrían una tendencia a presentar mayor Neuroticismo, Extraversión y Apertura, mientras que los rasgos Amabilidad y Responsabilidad se acentuarían con el paso de los años (Chopik y Kitayama, 2018; Lucas y Donnellan, 2011; Sánchez y Ledesma, 2013). Además, los cambios más significativos se observarían antes de alcanzar los 30 años de edad (Costa,
McCrae, y Löckenhoff, 2018; Schwaba y Bleidorn, 2017) y el tamaño del efecto de las diferencias que reportan los estudios suele ser pequeño (McCrae et al., 2000).

Específicamente en deportistas, González Hernández, Garcés de los Fayos Ruiz, y Ortega Toro (2014) analizaron diferencias en función del sexo y encontraron que las mujeres deportistas (de distintas disciplinas) puntuaban más alto en Neuroticismo, mientras que los hombres reportaban más Amabilidad y más Responsabilidad. No obstante, Cavallera, Passerini y Pepe (2013) evaluaron a un grupo de nadadores y encontraron que solo el rasgo Extraversión presentaba diferencias entre hombres y mujeres, siendo estas últimas quienes reportaron mayor presencia de ese rasgo. Otros estudios indican que los deportistas, sean hombres o mujeres, poseen rasgos de personalidad similares y que por tanto las diferencias en función del sexo son menores (Carazo Vargas y Araya Vargas, 2010; Garcés de Los Fayos Ruiz, Pérez Hernández-Ardieta, López María Dolores, 2002). Respecto a la edad, los estudios en deportistas sugieren resultados similares a los indicados para la población general: los deportistas adultos presentarían mayor Estabilidad emocional, Amabilidad y Responsabilidad que los más jóvenes (Rodríguez, 2003; Ruiz-Barquín y García-Naveira, 2013). Acerca del rasgo Apertura a la experiencia, García-Naveira, Ruiz-Barquín y Pujals (2011) y Ruiz-Barquín y García-Naveira (2013) encontraron que los futbolistas de alto nivel adultos tienen una mayor presencia de este rasgo que los jugadores cadetes y juveniles.

Otros estudios han indagado acerca de las diferencias en los rasgos de personalidad de los deportistas en función del nivel de competición (e.g., amateur vs. elite) o el nivel de experiencia de los mismos. Por ejemplo, Trninić, Trninić, y Penezić (2016) evaluaron a deportistas de equipo y encontraron que los jugadores senior re- 
portaban tener mayor Amabilidad y Responsabilidad que los que jugaban en ligas menores. Similarmente, Vaughan y Edwards (2020) encontraron que los sujetos con mayor experiencia deportiva reportaban también mayor Extraversión, Apertura y Responsabilidad, mientras que quienes tenían menor experiencia reportaron mayor Neuroticismo y mayor Amabilidad (los autores no especifican qué deporte practicaban los participantes). También Woodman, Zourbanos, Hardy, Beattie, y McQuillan (2010) reportan que los rasgos Responsabilidad y Estabilidad emocional se asocian con mejor calidad deportiva en gimnastas. En este sentido, la literatura sugiere que los rasgos de personalidad podrían asociarse con distintos niveles de rendimiento siendo especialmente las personas con mayor Responsabilidad las que presentan mayores posibilidades de éxito en los correspondientes deportes que practican (Habib et al., 2019; Mirzaei, Nikbakhsh, y Sharififar, 2013; Piedmont, Hill, y Blanco, 1999).

Respecto de las diferencias entre deportistas y población general, Hughes, Case, Stuempfle, y Evans (2003) analizaron los perfiles de los participantes de ultra-maratones y hallaron valores altos de Extraversión y Apertura a la experiencia en comparación con los valores normativos. García-Naveira et al. (2011), encontraron que los deportistas de alto nivel puntúan más alto en Extraversión y en Responsabilidad que quienes no hacen deportes. Según una revisión realizada por García-Naveira y Ruiz-Barquín (2013), los rasgos que caracterizan principalmente a los deportistas, en comparación con las personas no deportistas, son Extraversión, Estabilidad Emocional y Responsabilidad. Los autores agregan que Amabilidad y Apertura a la experiencia no suelen aparecer especialmente asociados a la actividad física. En Argentina, se encontraron diferencias significativas entre corredores de fondo y población general en los rasgos de Res- ponsabilidad y Neuroticismo, siendo los corredores quienes resultaron ser más responsables y más estables emocionalmente que la población general (Mortatti y Scapula, 2014; Sánchez, 2019). Los autores concluyen que ambos factores resultan necesarios para convertirse en un corredor experimentado, ya que no apegarse a los entrenamientos o desestabilizarse emocionalmente ante los contratiempos, por ejemplo, atentarían contra la permanencia de la persona en la disciplina.

Aunque existen variados estudios sobre deportes de gran difusión como el fútbol (e.g., García-Naveira et al., 2011), las carreras o maratones (e.g., Mortatti y Scápula, 2014) o el basketball (Marín et al, 2012), los estudios sobre deportes menos populares, como el judo, son escasos. Según Allen et al. (2013), los resultados de los diferentes estudios podrían no ser transferibles de manera directa entre las distintas disciplinas, por lo que resulta necesario realizar estudios específicos en cada deporte. Respecto al judo particularmente, los estudios sobre las características psicológicas implicadas en este deporte son sumamente escasos, aunque pueden destacarse los aportes de Ruiz-Barquín (2005, 2006, 2008, 2012). Acerca de las diferencias de sexo, Ruiz-Barquín (2005) realizó un estudio con judokas de ambos sexos, hallando que las judokas mujeres presentan puntuaciones más altas tanto en Neuroticismo como en Amabilidad. También Matsumoto, Takeuchi, Nakajima, y lida (2000) reportan un efecto moderador del sexo en el rendimiento de los judokas: el Neuroticismo predice el rendimiento en mujeres, mientras que la Amabilidad y la Apertura a la experiencia, hacen lo propio en varones. Acerca de la categoría de edad deportiva, Ruiz-Barquín (2005) indica que los judokas senior se describen como personas con mayor Estabilidad emocional (menos ansiosas, emotivas, impacientes o irritables), Amabilidad (más altruistas, cooperativos, amigables, cordiales, gene- 
rosos y empáticos) y Apertura (más cultos, informados e interesadas por experiencias y acontecimientos nuevos) en comparación con los judokas más jóvenes. Por su parte, Petar (2011) comparó la personalidad en judokas de élite y deportistas recreativos y encontró que los primeros reportaron menores niveles de Neuroticismo que los segundos. Acerca del rendimiento deportivo en judokas, los resultados son contradictorios: mientras que algunos resultados sugieren que los rasgos de personalidad que se asociarían con mayores victorias y mayores categorías de competición son Extraversión y Responsabilidad (Obmiński, Mroczkowska, y Tomaszewski, 2016; Ruíz-Barquín, 2006, 2008), otros indican también a los rasgos Amabilidad y Apertura a la experiencia como predictores (Ruiz-Barquín, 2005). Por último, acerca de las diferencias con población general o no deportistas, Ruiz-Barquín (2012) exploró los rasgos de deportistas de combate (judo, jiu-jitsu, karate, kenpo-karate, defensa personal, taekwondo, capoeira y esgrima) y de población general y encontró que, tanto en mujeres como varones, los niveles de Extraversión, Estabilidad emocional y Apertura a la experiencia eran mayores en los deportistas que en la población general.

En resumen, los estudios previos sugieren que existen relaciones entre la personalidad y distintos aspectos de la práctica deportiva. El estudio de los rasgos de personalidad en las distintas disciplinas parece especialmente importante si se considera que el estudio de estas variables puede contribuir a la detección de potenciales destrezas, habilidades, aptitudes y actitudes requeridas para un determinado deporte y, consecuentemente, favorece el desarrollo de intervenciones aplicadas en diversas áreas y contextos deportivos (Llames, Escudero, Marí, Vives, y Martí, 2017; Olmedilla, Sánchez-Aldeguer, Almansa, Gómez-Espejo, y Ortega, 2018; Ruiz-Barquín, 2006). Sin embargo, a pesar de que se han realizado variados estudios sobre deportes populares como el fútbol, los estudios al respecto de la disciplina deportiva del judo son escasos. Por tanto, el objetivo del presente estudio fue explorar los rasgos de personalidad de judokas senior estableciendo diferencias en función de (a) sexo, (b) edad, (c) graduación alcanzada, (d) nivel de competición, (e) años de experiencia deportiva, (f) categoría de peso, y (g) diferencias con población general.

\section{Método}

\subsection{Participantes}

Se trabajó con un muestreo intencional de 116 judokas de la categoría senior de Argentina, de los cuales, 86 fueron hombres (74.1\%), y 30 mujeres (25.9\%). Las edades estaban comprendidas entre los 15 y los 46 años $(M E=26.61 ; D T=6.85)$. Del total, 107 judokas (92.24\%) compitieron en las últimas tres temporadas. La media de años de experiencia en la disciplina fue de 17.3 años $(M d n=16.5 ; D T=7.92)$, con valores oscilando entre los 3 y los 38 años. Entre los atletas participantes, se encontraban medallistas de copas del mundo, de torneos panamericanos, sudamericanos, nacionales, y también, participantes de juegos olímpicos. La tabla 1 presenta características adicionales sobre los participantes del estudio. 
Tabla 1

Descripción detallada de la muestra

\begin{tabular}{|c|c|c|c|}
\hline Variables & Valores & $n$ & $\%$ \\
\hline \multirow{3}{*}{ Localización } & Provincia de Buenos Aires & 63 & $54.3 \%$ \\
\hline & Interior del país & 50 & $43.1 \%$ \\
\hline & Argentinos entrenando en el exterior & 3 & $2.6 \%$ \\
\hline \multirow{7}{*}{ Categoría peso } & $\begin{array}{c}\text { Superligero } \\
\text { (fem. }-48 \text { kg.; masc. }-60 \text { kg.) }\end{array}$ & 15 & $12.9 \%$ \\
\hline & $\begin{array}{c}\text { Semi-ligero } \\
\text { (fem. - } 52 \text { kg.; masc. }-66 \text { kg.) }\end{array}$ & 17 & $14.7 \%$ \\
\hline & $\begin{array}{c}\text { Ligero } \\
\text { (fem. }-57 \text { kg.; masc. }-73 \text { kg.) }\end{array}$ & 25 & $21.6 \%$ \\
\hline & $\begin{array}{c}\text { Semi-medio } \\
\text { (fem. -63 kg.; masc. }-81 \text { kg.) }\end{array}$ & 26 & $22.4 \%$ \\
\hline & $\begin{array}{c}\text { Medio } \\
\text { (fem. }-70 \mathrm{~kg} . ; \text { masc. }-90 \mathrm{~kg} .)\end{array}$ & 11 & $9.5 \%$ \\
\hline & $\begin{array}{c}\text { Semi-pesado } \\
\text { (fem. }-78 \text { kg.; masc. }-100 \text { kg.) }\end{array}$ & 15 & $12.9 \%$ \\
\hline & $\begin{array}{c}\text { Pesado } \\
\text { (fem. más de } 78 \mathrm{~kg} . ; \text { masc. más de 100kg.) }\end{array}$ & 7 & $6.0 \%$ \\
\hline \multirow{10}{*}{ Graduación } & 5to Kyu & 3 & $2.6 \%$ \\
\hline & 4to Kyu & - & - \\
\hline & 3er Kyu & 3 & $2.6 \%$ \\
\hline & 2do Kyu & 1 & $0.9 \%$ \\
\hline & 1er Kyu & 15 & $12.9 \%$ \\
\hline & 1er Dan & 62 & $53.4 \%$ \\
\hline & 2do Dan & 20 & $17.2 \%$ \\
\hline & 3er Dan & 6 & $5.2 \%$ \\
\hline & 4to Dan & 5 & $4.3 \%$ \\
\hline & 5to Dan & 1 & $0.9 \%$ \\
\hline \multirow{3}{*}{$\begin{array}{l}\text { Nivel máximo } \\
\text { de competición }\end{array}$} & Provincial & 1 & $0.9 \%$ \\
\hline & Nacional & 25 & $21.6 \%$ \\
\hline & Internacional & 90 & $77.6 \%$ \\
\hline
\end{tabular}

Se observa que 23 judokas eran Kyu (19.83\%), y $93(80.17 \%)$ eran Dan. Al respecto, los judokas poseen cinturones de color que representan sus conocimientos alcanzados y su crecimiento personal. Los cinturones básicos, denominados Kyu van desde el cinturón blanco (11vo $K y u$ ), hasta el cinturón marrón (1er Kyu), pasando por 9 niveles intermedios. Luego de completados los niveles básicos de los $\mathrm{Kyu}$, se accede a la categoría de los Dan, que habilita la enseñanza de la disciplina. La categoría Dan posee 5 niveles de cinturón negro y 5 niveles de Maestro, es decir, un total de 10 niveles. Los 6 to, $7 \mathrm{mo}$ y $8 \mathrm{vo}$ Dan son representados por cinturones de Maestro de color intercalado rojo-blanco, mientras que el 9 no y 10 mo Dan, son las dos máximas graduaciones y su color distintivo es el rojo liso. 


\subsection{Medidas}

Listado de Adjetivos para Evaluar la Personalidad: para evaluar los rasgos de personalidad se aplicó el Listado de Adjetivos para Evaluar la Personalidad -AEP- (Ledesma et al., 2011; Sánchez y Ledesma, 2007, 2013), el cual es un instrumento autoadministrable que consiste en 67 adjetivos descriptores de rasgos, basado en el MCF de Costa y McCrae. Se optó por utilizar este instrumento puesto que se basa en el ampliamente difundido MCF y es significativamente más breve que otros instrumentos de evaluación de la personalidad (e.g., Big Five Questionnaire, Bermúdez, 1995; Test Personalidad de TEA, Corral Gregorio, Pamos de la Hoz, Pereña Brand, y Seisdedos Cubero, 2009), requiriendo aproximadamente entre $10 \mathrm{y}$ 15 minutos para ser completado. Además, resulta de fácil comprensión y administración y fue desarrollado en el contexto argentino y validado en una amplia muestra (Amabilidad: $\alpha$ entre .75 y .84; Neuroticismo: $\alpha$ entre .73 y .85; Responsabilidad: $\alpha$ entre .73 y .80; Extraversión: $\alpha$ entre .79 y .85; Apertura a la experiencia: $\alpha$ entre .72 y .77; Sanchez y Ledesma, 2007; Ledesma et al., 2011), por lo que contempla las características culturales y lingüísticas del país. En el AEP, los participantes deben contestar cada ítem indicando cuánto lo describe ese adjetivo. El formato de respuesta es de tipo Likert, desde 1 ("no me describe en absoluto") hasta 5 ("me describe tal cual soy"). Cada factor (Amabilidad/Antagonismo, Responsabilidad//rresponsabilidad, Extraversión/Introversión, Neuroticismo/Estabilidad Emocional y Apertura a la Experiencia/Cerrado a la Experiencia) provee un valor obtenido a partir del promedio de los adjetivos que corresponden a cada dimensión, invirtiendo los ítems que así lo requieran. De esta forma, se consigue un valor entre 1 y 5 para cada escala, resultando una mayor presencia del rasgo cuanto mayor sea el resultado en la escala evaluada. En el presente es- tudio, los valores a fueron .65 para Apertura a la experiencia, .79 para Extraversión, .78 para Responsabilidad, .86 para Neuroticismo y .81 Amabilidad. Acerca de la muestra normativa de baremación del AEP (Sánchez y Ledesma, 2013) con la que se compararon los rasgos de personalidad de los judokas, se trata de una muestra no probabilística compuesta por 1680 participantes de población general argentina, con edades comprendidas entre 18 y 89 años (ME: 36.8; DT: 18.6), de los cuales 1149 eran mujeres $(68.4 \%)$ y 524 hombres (31.2\%); de 7 de los participantes $(0.4 \%)$ no se obtuvo este dato.

Datos sociodemográficos y deportivos: se recabaron datos personales, demográficos y deportivos de los judokas participantes en la investigación. Se indagó acerca de la edad, el sexo, la ciudad de residencia, la ciudad de proveniencia, la graduación (especificando cinturón máximo alcanzado), la categoría actual en la que compite, la categoría de peso en la que compite, los años de experiencia en el judo, el máximo nivel de competición alcanzado (provincial, nacional, internacional) y el tiempo transcurrido desde su última competición.

\subsection{Procedimiento}

Se trató de un estudio de metodología cuantitativa, de tipo exploratorio-descriptivo, con un diseño no experimental, transversal (Hernández Sampieri, Fernández Collado, y Baptista Lucio, 2014). Para contactar a los distintos judokas senior del país, se relevaron los datos de contacto de los ganadores de los podios argentinos de judo de los torneos nacionales Apertura y Clausura de los tres años previos a la recolección de datos de presente estudio. Se utilizó la información disponible en las federaciones provinciales y en los dojos del país. A su vez, algunos de dichos judokas difundieron el estudio y pusieron a los investigadores en contac- 
to con otros competidores senior locales que conocían. Se contactó a los judokas vía internet, se les informó acerca de las características del estudio y se confirmó su participación voluntaria y confidencial mediante la firma de un consentimiento informado digital. Los protocolos auto-administrados fueron completados por medio de la plataforma de Google forms, con un cuestionario creado para tal fin. En todo momento, se siguieron todas las normativas para la investigación en seres humanos recomendadas por la American Psychological Association (APA, 2010) y la Declaración de Helsinki (World Medical Association, 2013).

\subsection{Análisis De Datos}

Los datos cuantitativos fueron sistematizados e ingresados en una única base informatizada. Para caracterizar a la muestra bajo estudio se aplicaron análisis estadísticos descriptivos. Se comprobó la normalidad de la distribución de los cinco rasgos de personalidad siguiendo el criterio que establece que valores de asimetría y curtosis comprendidos entre \pm 2 puntos se consideran como límites aceptables de dicha distribución (e.g., Field, 2009; George y Mallery, 2016; Trochim y Donnelly, 2006). Para determinar la existencia de diferencias en función del sexo, se aplicaron pruebas $U$ de Mann-Whitney. Se optó por un estadístico no paramétrico por ser más robusto frente a las diferencias en los tamaños de ambos grupos y puesto que algunos de los análisis revelaron incumplimiento del criterio de homocedasticidad (prueba de Levene: $p>$ .01). Para estimar el tamaño del efecto de las diferencias se calculó la $d$ de Cohen, siguiendo los criterios establecidos por Cohen (1988) para estimar la magnitud de las diferencias (pequeñas: 0.30; medianas: 0.50; grandes: 0.80).

Para determinar si existían diferencias en función de la edad de los participantes, y considerando que estudios previos sugie- ren que cerca de los 30 años se observa una mayor estabilidad interindividual en los rasgos de personalidad (Costa et al., 2018; Ledesma et al., 2011; Schwaba y Bleidorn, 2017), se dividió a los participantes entre aquellos que indicaron tener hasta 29 años o menos $(n=79)$, y aquellos que indicaron tener 30 años o más ( $n$ = 37). Ante el incumplimiento de la prueba de Levene $(p>.01)$, se aplicaron pruebas $U$ de Mann-Whitney para determinar la existencia de diferencias entre los grupos. También se calcularon los valores estadísticos descriptivos (media, desviación típica) para cada grupo y se calculó la $d$ de Cohen para estimar la magnitud de las diferencias.

Para analizar el efecto de la graduación, se dividió a los judokas en dos grupos: por un lado, aquellos que se encontraban en la categoría Kyu o en el primer Dan y, por otro lado, aquellos judokas que ya habían alcanzado categorías superiores del Dan (2do, 3ro, 4to y 5to). Se aplicaron pruebas $U$ de Mann-Whitney en consideración al tamaño de los grupos y a que los rasgos Neuroticismo y Apertura a la experiencia no cumplieron con el criterio de homocedasticidad (Levene: $p>.01$ ). También se calcularon los valores estadísticos descriptivos (media, desviación típica) para cada grupo y la $d$ de Cohen en los casos en los que existían diferencias significativas.

Para evaluar el efecto del nivel máximo de competición alcanzado, se consideraron las diferencias entre los judokas con nivel nacional e internacional (solo un judoka indicó que su nivel máximo era el provincial, por lo que no fue considerado en este análisis). Se aplicaron para este propósito pruebas $U$ de Mann-Whitney en consideración al tamaño de los grupos y a que el rasgo Amabilidad no cumplió con el criterio de homocedasticidad (Levene: $p>$ .01). También se calcularon los valores estadísticos descriptivos (media, desviación típica) para cada grupo y la $d$ de Cohen en 
los casos en los que existían diferencias significativas.

Con el objeto de analizar el efecto de los años de experiencia deportiva, se dividió a los participantes en dos grupos en función de la mediana de años practicando la disciplina (16.5). El primer grupo quedó así conformado por quienes indicaron tener 16 años de experiencia o menos, y quienes indicaron tener 17 años de experiencia o más. En este caso, se aplicaron pruebas $t$ de Student para muestras independientes, puesto que el tamaño de los grupos fue equivalente $(n=68)$ y las pruebas de Levene revelaron homocedasticidad para todas las variables $(p>.05)$. También se calcularon los valores estadísticos descriptivos (media, desviación típica) para cada grupo y la $d$ de Cohen en los casos en los que existían diferencias significativas.

Para estimar si existían diferencias en los rasgos de personalidad según la categoría de peso en la cual competían los judokas, se dividió a los participantes en tres grupos en función de su pertenencia a las categorías ligeras (peso super-ligero, semi-ligero y ligero), medias (peso semi-medio y medio) o pesadas (peso se- mi-pesado y pesado). Luego, se aplicaron pruebas ANOVA de un factor (Levene; $p>$ .05). También se calcularon los valores estadísticos descriptivos (media, desviación típica) para cada grupo.

Finalmente, se procedió a evaluar si existían diferencias entre los rasgos de personalidad de los judokas y los de la población normotípica. Se realizaron comparaciones por medio de pruebas $t$ de Student para una muestra, considerando como valores de referencia los obtenidos por Sánchez y Ledesma (2013) en su estudio en población general. Dado que los baremos del instrumento AEP distinguen entre hombres y mujeres y los resultados del presente estudio también evidenciaron diferencias en función del sexo, los análisis se realizaron por separado para estos dos grupos. Se calculó también el tamaño del efecto ( $d$ de Cohen) para aquellos rasgos que mostraron diferencias.

\section{Resultados}

Para caracterizar a la muestra en función de los rasgos de personalidad se aplicaron análisis estadísticos descriptivos. Los resultados de presentan para toda la muestra en la tabla 2 para la muestra total.

Tabla 2

Valores estadísticos descriptivos para la muestra total

\begin{tabular}{|l|c|c|c|c|c|c|}
\hline & Mín. & Máx. & ME & DT & Asimetría & Curtosis \\
\hline Extraversión & 2.20 & 4.90 & 3.74 & 0.62 & -0.56 & -0.16 \\
\hline Amabilidad & 2.69 & 5.00 & 4.04 & 0.44 & -0.53 & 0.62 \\
\hline Responsabilidad & 2.08 & 4.92 & 3.84 & 0.54 & -0.38 & 0.31 \\
\hline Neuroticismo & 1.44 & 4.17 & 2.60 & 0.63 & 0.46 & -0.30 \\
\hline Apertura & 2.40 & 4.90 & 3.45 & 0.52 & 0.41 & -0.17 \\
\hline
\end{tabular}

Para determinar la existencia de diferencias en función del sexo, se aplicaron pruebas $U$ de Mann-Whitney. También se calcularon los valores estadísticos descriptivos diferenciados por sexo y se estimó el tamaño del efecto de las diferencias significativas ( $d$ de Cohen). Los resultados se presentan en la Tabla 3. El único rasgo de Personalidad que evidenció diferencias estadísticamente significativas en función del sexo fue Neuroticismo, siendo este mayor en las mujeres que en los hombres. 
El tamaño del efecto de esta diferencia fue medio. El resto de los rasgos no registró diferencias significativas entre hombres y mujeres.

Tabla 3

Valores estadísticos descriptivos de los rasgos de personalidad diferenciados por sexo, pruebas U de Mann-Whitney y tamaño del efecto

\begin{tabular}{|l|c|c|c|c|c|c|c|c|}
\hline & \multicolumn{2}{|c|}{ Femenino } & \multicolumn{2}{c|}{ Masculino } & \multirow{2}{*}{$U$} & \multirow{2}{*}{$\mathrm{Z}$} & \multirow{2}{*}{$p$} & $\begin{array}{c}d \text { de } \\
\text { Cohen }\end{array}$ \\
\hline & ME & DT & ME & DT & & & & - \\
\hline Extraversión & 3.74 & 0.51 & 3.73 & 0.66 & 1265.000 & -0.16 & .88 & - \\
\hline Amabilidad & 4.04 & 0.33 & 4.05 & 0.47 & 1266.000 & -0.15 & .88 & - \\
\hline Responsabilidad & 3.87 & 0.46 & 3.83 & 0.57 & 1250.000 & -0.25 & .80 & - \\
\hline Neuroticismo & 2.89 & 0.62 & 2.50 & 0.61 & 828.000 & -2.98 & $<.01$ & 0.63 \\
\hline $\begin{array}{l}\text { Apertura a la } \\
\text { Experiencia }\end{array}$ & 3.46 & 0.46 & 3.44 & 0.54 & 1246.000 & -0.28 & .78 & - \\
\hline
\end{tabular}

Para determinar si existían diferencias en función de la edad de los judokas, se dividió a la muestra entre quienes tenían 29 años o menos y quienes indicaron tener 30 años o más. Se aplicaron pruebas $U$ de Mann-Whitney y se estimó el tamaño del efecto de las diferencias significativas
( $d$ de Cohen). Los resultados se presentan en la Tabla 4 y muestran que los judokas de mayor edad reportaron mayor Amabilidad, Responsabilidad y menor Neuroticismo. Las diferencias resultaron pequeñas a moderadas.

\section{Tabla 4}

Valores estadísticos descriptivos de los rasgos de personalidad diferenciados por grupo de edad, pruebas $U$ de Mann-Whitney y tamaño del efecto

\begin{tabular}{|l|c|c|c|c|c|c|c|c|}
\hline & \multicolumn{2}{|c|}{$\begin{array}{c}29 \text { años o } \\
\text { menos }\end{array}$} & \multicolumn{2}{l|}{30 años o más } & \multirow{2}{*}{$U$} & \multirow{2}{*}{$Z$} & $p$ & $\begin{array}{c}d \text { de } \\
\text { Cohen }\end{array}$ \\
\hline & $\mathrm{ME}$ & $\mathrm{DT}$ & $\mathrm{ME}$ & $\mathrm{DT}$ & & & & \\
\hline Extraversión & 3.75 & 0.62 & 3.70 & 0.63 & 1375.000 & -0.51 & .61 & - \\
\hline Amabilidad & 3.97 & 0.43 & 4.20 & 0.42 & 1022.500 & -2.61 & $<.01$ & -0.54 \\
\hline Responsabilidad & 3.76 & 0.51 & 4.01 & 0.58 & 999.500 & -2.74 & $<.01$ & -0.46 \\
\hline Neuroticismo & 2.68 & 0.66 & 2.44 & 0.55 & 1116.500 & -2.05 & .04 & 0.40 \\
\hline $\begin{array}{l}\text { Apertura a la } \\
\text { Experiencia }\end{array}$ & 3.39 & 0.47 & 3.56 & 0.59 & 1291.500 & -1.01 & .31 & - \\
\hline
\end{tabular}

Para analizar el efecto de la graduación, se dividió a los judokas en dos grupos: por un lado, aquellos que se encontraban en la categoría Kyu o en el primer Dan y, por otro lado, aquellos judokas que ya habían alcanzado categorías superiores del Dan (2do, 3ro, 4to y 5to). Los resultados de las pruebas $U$ de Mann-Whitney, los estadísticos descriptivos para cada grupo y el tamaño del efecto de las diferencias se presentan en la Tabla 5. Se encontró una diferencia estadísticamente significativa y moderada en el rasgo Neuroticismo, presentando los competidores de menores graduaciones mayores valores que los de graduaciones más altas. El resto de los rasgos de personalidad no mostró diferencias en función del grupo de graduación. 


\section{Tabla 5}

\section{Valores estadísticos descriptivos de los rasgos de personalidad por grupo de graduación, pruebas U de Mann-Whitney y tamaño del efecto}

\begin{tabular}{|c|c|c|c|c|c|c|c|c|}
\hline & \multicolumn{2}{|c|}{$\begin{array}{c}\text { 5to a 1er Kyu y } \\
\text { 1er Dan }\end{array}$} & \multicolumn{2}{|c|}{$\begin{array}{l}\text { 2do, 3er, 4to y } \\
5 \text { to Dan }\end{array}$} & \multirow[t]{2}{*}{$U$} & \multirow[t]{2}{*}{ Z } & \multirow[t]{2}{*}{$p$} & \multirow{2}{*}{$\begin{array}{l}d \text { de } \\
\text { Cohen }\end{array}$} \\
\hline & ME & DT & ME & DT & & & & \\
\hline Extraversión & 3.71 & 0.63 & 3.81 & 0.60 & 1204.500 & -0.86 & .39 & - \\
\hline Amabilidad & 4.00 & 0.46 & 4.16 & 0.38 & 1133.500 & -1.30 & .19 & - \\
\hline Responsabilidad & 3.79 & 0.56 & 3.99 & 0.49 & 1070.500 & -1.69 & .09 & - \\
\hline Neuroticismo & 2.70 & 0.67 & 2.35 & 0.44 & 919.500 & -2.62 & $<.01$ & 0.62 \\
\hline $\begin{array}{l}\text { Apertura a la } \\
\text { Experiencia }\end{array}$ & 3.42 & 0.48 & 3.52 & 0.60 & 1218.500 & -0.78 & .44 & - \\
\hline
\end{tabular}

Para evaluar el efecto del nivel máximo de competición alcanzado (nacional e internacional) se aplicaron pruebas $U$ de Mann-Whitney y se calcularon los estadísticos descriptivos de cada grupo de judokas y el tamaño del efecto de las diferencias. Los resultados de las pruebas descriptivas e inferenciales se presentan en la Tabla 6. Puede observarse que el rasgo Apertura a la experiencia presentó una diferencia estadísticamente significativa moderada, siendo los competidores de nivel internacional quienes evidenciaron mayores valores en dicho rasgo en comparación los competidores de nivel nacional. El resto de los rasgos de personalidad no evidenció diferencias en función de esta variable.

Tabla 6

Valores estadísticos descriptivos de los rasgos de personalidad por nivel máximo de competición, pruebas $U$ de Mann-Whitney y tamaño del efecto

\begin{tabular}{|c|c|c|c|c|c|c|c|c|}
\hline & \multicolumn{2}{|c|}{$\begin{array}{c}\text { Nivel } \\
\text { internacional }\end{array}$} & \multicolumn{2}{|c|}{ Nivel nacional } & \multirow[t]{2}{*}{$U$} & \multirow[t]{2}{*}{ Z } & \multirow{2}{*}{$p$} & \multirow{2}{*}{$\begin{array}{c}d \text { de } \\
\text { Cohen }\end{array}$} \\
\hline & $\mathrm{ME}$ & DT & $\mathrm{ME}$ & DT & & & & \\
\hline Extraversión & 3.76 & 0.64 & 3.66 & 0.54 & 977.500 & -1.00 & .32 & - \\
\hline Amabilidad & 4.02 & 0.47 & 4.15 & 0.30 & 940.500 & -1.25 & .21 & - \\
\hline Responsabilidad & 3.85 & 0.54 & 3.79 & 0.57 & 1034.000 & -0.62 & .54 & - \\
\hline Neuroticismo & 2.60 & 0.63 & 2.62 & 0.66 & 1076.500 & -0.33 & .74 & - \\
\hline $\begin{array}{l}\text { Apertura a la } \\
\text { Experiencia }\end{array}$ & 3.50 & 0.52 & 3.27 & 0.48 & 769.500 & -2.42 & .02 & 0.46 \\
\hline
\end{tabular}

Con el objeto de analizar si existían diferencias en función de los años de experiencia deportiva, se dividió a los participantes entre aquellos que poseían 16 años de experiencia deportiva o menos, y quienes poseían 17 años o más. En este caso, se aplicaron pruebas $t$ de Student para muestras independientes. También se calcularon los estadísticos descriptivos y el tamaño del efecto de las diferencias. Los resultados se presentan en la Tabla 7 y muestran una diferencia de tamaño medio en el rasgo Apertura a la experiencia, siendo los judokas con más años de experiencia deportiva los que reportan mayor presencia de este rasgo. 
Rasgos de personalidad en judokas senior y diferencias con población general

Tabla 7

Valores estadísticos descriptivos de los rasgos de personalidad por grupo de años de experiencia deportiva, pruebas t de Student, Levene y tamaño del efecto

\begin{tabular}{|c|c|c|c|c|c|c|c|c|c|}
\hline & \multicolumn{2}{|c|}{$\begin{array}{l}\text { Experiencia: } 16 \\
\text { años o menos }\end{array}$} & \multicolumn{2}{|c|}{$\begin{array}{c}\text { Experiencia: } \\
17 \text { años o más }\end{array}$} & \multirow{2}{*}{$\begin{array}{l}\text { Levene } \\
(p)\end{array}$} & \multirow[t]{2}{*}{$t$} & \multirow[t]{2}{*}{$p$} & \multirow{2}{*}{$\begin{array}{l}\text { Dif. de } \\
\text { medias }\end{array}$} & \multirow{2}{*}{$\begin{array}{l}d \text { de } \\
\text { Cohen }\end{array}$} \\
\hline & ME & DT & $\mathrm{ME}$ & DT & & & & & \\
\hline Extraversión & 3.70 & 0.64 & 3.77 & 0.60 & $0.01(.97)$ & -0.61 & .54 & -0.07 & - \\
\hline Amabilidad & 3.99 & 0.44 & 4.10 & 0.44 & $0.23(.63)$ & -1.28 & .20 & -0.10 & - \\
\hline $\begin{array}{l}\text { Responsabi- } \\
\text { lidad }\end{array}$ & 3.76 & 0.55 & 3.92 & 0.53 & $0.59(.44)$ & -1.64 & .10 & -0.16 & - \\
\hline Neuroticismo & 2.63 & 0.68 & 2.58 & 0.58 & $1.81(.18)$ & 0.42 & .68 & 0.05 & - \\
\hline $\begin{array}{l}\text { Apertura a la } \\
\text { Experiencia }\end{array}$ & 3.34 & 0.47 & 3.56 & 0.54 & $2.47(.12)$ & -2.33 & .02 & -0.22 & -0.43 \\
\hline
\end{tabular}

A continuación, para estimar si existían diferencias en los rasgos de personalidad según la categoría de peso en la cual competían los judokas (ligeras, medias, pesadas) se aplicaron pruebas ANOVA de un factor. Los resultados (Tabla 8) mostraron ausencia de diferencias estadísticamente significativas en los rasgos de personalidad en función de las categorías de peso en la cual los judokas compiten.

Tabla 8

Valores estadísticos descriptivos de los rasgos de personalidad por categoría de peso, pruebas ANOVA y Levene

\begin{tabular}{|l|c|c|c|c|c|c|c|c|c|}
\hline & \multicolumn{2}{|c|}{ Pesos ligeros } & Pesos medios & \multicolumn{2}{c|}{$\begin{array}{c}\text { Pesos } \\
\text { pesados }\end{array}$} & $\begin{array}{c}\text { Levene } \\
(p)\end{array}$ & $F$ & $p$ \\
\hline & $\mathrm{ME}$ & $\mathrm{DT}$ & $\mathrm{ME}$ & $\mathrm{DT}$ & $\mathrm{ME}$ & $\mathrm{DT}$ & & & \\
\hline Extraversión & 3.74 & 0.55 & 3.85 & 0.59 & 3.53 & 0.78 & $1.44(.24)$ & 1.89 & .16 \\
\hline Amabilidad & 4.04 & 0.44 & 4.02 & 0.48 & 4.10 & 0.39 & $0.14(.87)$ & 0.23 & .79 \\
\hline Responsabilidad & 3.88 & 0.50 & 3.82 & 0.60 & 3.76 & 0.56 & $0.20(.82)$ & 0.43 & .65 \\
\hline Neuroticismo & 2.64 & 0.66 & 2.62 & 0.66 & 2.46 & 0.52 & $1.10(.34)$ & 0.71 & .49 \\
\hline $\begin{array}{l}\text { Apertura a la } \\
\text { Experiencia }\end{array}$ & 3.46 & 0.53 & 3.41 & 0.49 & 3.49 & 0.54 & $0.12(.89)$ & 0.20 & .82 \\
\hline
\end{tabular}

Finalmente, se procedió a evaluar si existían diferencias entre los rasgos de personalidad de los judokas y los de la población normotípica de referencia (Sánchez y Ledesma, 2013), considerando por separado hombres y mujeres. Se calculó también el tamaño del efecto ( $d$ de Cohen) para aquellos rasgos que mostraron diferencias y se calcularon los estadísticos descriptivos. Los resultados se expresan en la Tabla 9 para las mujeres y en la Tabla 10 para los hombres. Se observaron diferencias entre las mujeres judokas y la po- blación general en el rasgo Apertura a la Experiencia, siendo la diferencia entre pequeña y moderada a favor de las deportistas. En cuanto a los judokas masculinos, se hallaron diferencias pequeñas para los rasgos Amabilidad y Responsabilidad siendo los judokas quienes reportan una presencia más marcada de estos rasgos. También se encontró una diferencia moderada para el rasgo Neuroticismo, siendo en este caso los judokas quienes presentan valores inferiores. 
Tabla 9

Tamaño del efecto de la diferencias de medias entre la muestra de judokas mujeres y la muestra de población general de mujeres.

\begin{tabular}{|l|c|c|c|c|c|c|c|c|}
\hline \multirow{2}{*}{} & \multicolumn{2}{|c|}{$\begin{array}{c}\text { Judokas } \\
\text { mujeres }\end{array}$} & \multicolumn{2}{c|}{$\begin{array}{c}\text { Mujeres población } \\
\text { general }\end{array}$} & \multirow{2}{*}{$t$} & $p$ & $\begin{array}{c}\text { Dif. de } \\
\text { medias }\end{array}$ & $\begin{array}{c}d \text { de } \\
\text { Cohen }\end{array}$ \\
\cline { 2 - 8 } & $\mathrm{M}$ & $\mathrm{DT}$ & $\mathrm{M}$ & $\mathrm{DT}$ & & & & - \\
\hline Extraversión & 3.76 & 0.47 & 3.77 & 0.67 & -0.32 & .75 & -0.03 & - \\
Amabilidad & 4.04 & 0.33 & 4.01 & 0.49 & 0.49 & .63 & 0.03 & - \\
Responsabilidad & 3.87 & 0.46 & 3.74 & 0.61 & 1.50 & .15 & 0.13 & - \\
Neuroticismo & 2.89 & 0.62 & 3.01 & 0.60 & -1.11 & .28 & -1.12 & - \\
$\begin{array}{l}\text { Apertura a la } \\
\text { Experiencia }\end{array}$ & 3.46 & 0.46 & 3.22 & 0.68 & 2.83 & $<.01$ & 0.24 & 0.41 \\
\hline
\end{tabular}

Tabla 10

Tamaño del efecto de la diferencias de medias entre la muestra de judokas varones y la muestra de población general de varones.

\begin{tabular}{|l|c|c|c|c|c|c|c|c|}
\hline & \multicolumn{2}{|c|}{$\begin{array}{c}\text { Judokas } \\
\text { hombres }\end{array}$} & \multicolumn{2}{c|}{$\begin{array}{c}\text { Hombres población } \\
\text { general }\end{array}$} & $t$ & $p$ & $\begin{array}{c}\text { Dif. de } \\
\text { medias }\end{array}$ & $\begin{array}{c}d \text { de } \\
\text { Cohen }\end{array}$ \\
\hline & $\mathrm{ME}$ & $\mathrm{DT}$ & $\mathrm{ME}$ & $\mathrm{DT}$ & & & & \\
\hline Extraversión & 3.71 & 0.69 & 3.68 & 0.67 & 0.76 & .45 & 0.05 & - \\
\hline Amabilidad & 4.05 & 0.47 & 3.93 & 0.51 & 2.26 & .03 & 0.12 & 0.24 \\
\hline Responsabilidad & 3.83 & 0.57 & 3.67 & 0.64 & 2.63 & $<.01$ & 0.16 & 0.26 \\
\hline Neuroticismo & 2.50 & 0.61 & 2.79 & 0.57 & -4.36 & $<.01$ & -0.42 & -0.49 \\
\hline $\begin{array}{l}\text { Apertura a la } \\
\text { Experiencia }\end{array}$ & 3.44 & 0.54 & 3.36 & 0.65 & 1.44 & .15 & 0.08 & - \\
\hline
\end{tabular}

\section{Discusión}

El presente estudio tuvo por objetivo explorar los rasgos de personalidad de judokas senior estableciendo diferencias en función de (a) sexo, (b) edad, (c) graduación alcanzada, (d) nivel de competición, (e) años de experiencia deportiva, (f) categoría de peso, y $(\mathrm{g})$ diferencias con población general. En principio, respecto de las diferencias de sexo en los rasgos de personalidad, se encontraron diferencias para el rasgo Neuroticismo, siendo más elevado este en mujeres que en hombres. Esto es semejante a lo expuesto en otras investigaciones sobre las diferencias en el rasgo Neuroticismo población general (e.g., Barra Almagiá et al., 2019; Church, 2016; Costa et al., 2001; De Miguel Negredo, 2005; Langvik et al., 2016; Sánchez y Ledesma, 2013; Schmitt et al., 2008, 2017; Weisberg et al., 2011), e indica que las mujeres parecerían poseer mayores niveles de ansiedad, impulsividad, tensión $e$ inestabilidad emocional $y$, en consecuencias, los hombres serían menos proclives a vivenciar emociones negativas, serían menos ansiosos, irritables o emotivos. También es similar a lo reportado por González Hernández et al. (2014) sobre diferencias en los niveles de Neuroticismo entre hombres y mujeres deportistas, así como a los resultados obtenidos por Ruiz-Barquín (2005) en judokas específicamente. El resto de los rasgos del MCF no evidenciaron diferencias estadísticamente significativas en función del sexo. Este hallazgo, difiere de lo expresado en la literatura (e.g. De Miguel Negredo, 2005; Schmitt et al., 2008; Schmitt et al., 
2017; Weisberg et al., 2011), siendo que, en general, suelen encontrarse diferencias entre hombres y mujeres para el resto de los rasgos del MCF, tanto en población general (Barra Almagiá et al., 2019; Costa et al., 2001; Sánchez y Ledesma, 2013; Schmitt et al., 2017), como en deportistas (Cavallera et al., 2013; González Hernández et al., 2014). Es probable es que estas discrepancias puedan deberse a las características de los atletas en sí mismas, es decir, a que las características de personalidad de quienes practican deportes se asemejen entre sí, minimizando las diferencias que comúnmente se atribuyen al sexo en la población general, lo cual ha sido sugerido por otros autores (Carazo Vargas y Araya Vargas, 2010; Garcés de Los Fayos Ruiz et al., 2002). En este sentido, Ruíz Barquín (2005), también halló inexistencia de diferencias entre hombres y mujeres judokas en los rasgos de Responsabilidad, Apertura a la Experiencia y Extraversión -aunque sí reporta diferencias para Amabilidad-.

Respecto a la edad, los resultados mostraron que los judokas de más de 30 años reportaban mayor Amabilidad, Responsabilidad y Estabilidad emocional, lo cual es similar a lo encontrado en otros estudios (Chopik y Kitayama, 2018; Lucas y Donnellan, 2011; Sánchez y Ledesma, 2013) y sugiere que estos rasgos se acentuarían con el paso de los años. Esto también coincide con los hallazgos en deportistas adultos, quienes presentan más Estabilidad emocional, Amabilidad y Responsabilidad que los más jóvenes (Rodríguez, 2003; Ruiz-Barquín y García-Naveira, 2013), y con lo hallado por Ruiz-Barquín (2005) en judokas.

Respecto a la indagación de las diferencias en función de la graduación de los judokas, se encontraron menores niveles de Neuroticismo en mayores niveles de graduación. Esto es similar a lo reportado por Ruiz-Barquín (2005) acerca de las ca- tegorías, donde el autor concluye que los judokas hombres de la categoría senior se describen a sí mismo como menos ansiosos, emotivos, irritables e impacientes que sus pares de categorías inferiores. No obstante, dicho estudio también reporta mayor Responsabilidad, Amabilidad y Apertura a la Experiencia en los judokas senior en comparación con otras categorías juveniles. Es posible que diferencias a nivel cultural entre España y Argentina así como la consideración de la variable edad (Ledesma et al., 2011), expliquen estas discrepancias. Además, cabe destacar que el presente estudio trabajó únicamente con judokas senior y, por tanto, la agrupación por medio de categorías de competición no es equivalente a la agrupación por la graduación.

En el presente estudio también se encontró que aquellos competidores que habían alcanzado el nivel internacional presentaban mayores niveles de Apertura a la experiencia que aquellos cuyo nivel máximo era el nacional. Similarmente, también se encontró que aquellos judokas que poseían más años de experiencia en la disciplina (i.e., 17 años o más) presentaban también mayores niveles de Apertura. Si bien en la literatura no se detectaron investigaciones que hayan tenido en cuenta estas variables específicas para esta población, Ruiz-Barquín (2005) indicó que los judokas con mayores categorías, se describían a sí mismos como personas más cultas e interesadas por experiencias y acontecimientos nuevos, que los judokas de menores categorías. Es posible pensar que los judokas con preferencia por lo nuevo y lo diferente, y menos gusto por la rutina y lo predecible, hayan perseguido el interés por alcanzar un mayor nivel y competir en otros países a nivel internacional, enfrentando así mayor novedad y diversidad (i.e., hipótesis de selección; García-Naveira, 2010). También es posible pensar que la participación en la práctica deportiva de mayor nivel es uno de los 
factores que contribuye al desarrollo de la personalidad, específicamente a mayor Apertura a la experiencia o (i.e., hipótesis de desarrollo).

Respecto a la ausencia de diferencias en función de la categoría de peso en la cual los distintos judokas compiten, no se han encontrado estudios previos al respecto que permitan comparar los hallazgos. Es posible pensar que los rasgos de personalidad de los judokas sean independientes del rango de peso en el cual compiten, y que las diferencias interindividuales referidas a las variables antes analizadas (i.e., sexo, edad, experiencia, etc.) al interior de las categorías de peso sean factores de mayor consideración.

Respecto de las diferencias con población general, la información obtenida de los análisis parece sugerir que los judokas hombres poseen mayor Amabilidad, Responsabilidad, mayor Estabilidad Emocional que la población general, mientras que en las judokas mujeres se destaca la Apertura a la Experiencia. Sobre la Estabilidad Emocional, es posible que la disciplina fortalezca la capacidad de no actuar por impulso, de tener clara la mente, de sentirse seguro y de ser capaz de controlar distintas situaciones. Similarmente, los niveles altos de Responsabilidad serían importantes para cumplir con el deporte y mantener la perseverancia, la voluntad y el sacrificio para cumplir con los objetivos. Al respecto, tanto García-Naveira et al. (2011), como Mortatti y Scápula (2014) reportaron resultados similares respecto de las diferencias entre deportistas y población general, concluyendo que los deportistas son más estables emocionalmente y más responsables. De manera similar, Ruiz-Barquín (2012) halló mayor prevalencia de Estabilidad Emocional en deportistas de combate que en población general. También Rhodes y Smith (2006) sugieren que la Responsabilidad y la Estabilidad Emocional estarían positivamente asociadas con la actividad física. En este sentido, los deportes de alto rendimiento requerirían mayores compromisos por parte de la persona, conllevando en mayor acentuación de dichos rasgos.

Respecto al rasgo Amabilidad (ser respetuoso, tener compañerismo, ser tranquilo, pensar en el otro, ser empático) el cual registró niveles más altos entre los judokas hombres que entre la población general, las diferencias coinciden con investigaciones que hallaron mayores niveles de Amabilidad en deportistas de combate que en población general (e.g., Ruiz-Barquín, 2012), aunque difiere de los indicado por García-Naveira y Ruiz-Barquín (2013), quienes afirman que el rasgo Amabilidad, no suele aparecer especialmente asociado a la actividad física en general. Así, es posible que sean los deportes de combate los que implican con mayor frecuencia a este rasgo, aunque nuevos estudios deberán realizarse para esclarecer estas discrepancias.

Asimismo, respecto del rasgo Apertura a la Experiencia, el mismo presentó diferencias solo para las judokas mujeres. Ruiz-Barquín (2012) también reporta mayor Apertura a la experiencia en deportistas de combate que en población general y sugiere que esto no es extraño puesto que dicho rasgo se encuentra asociado con el aprendizaje de nuevas habilidades y la orientación hacia la búsqueda de nuevas de experiencias. Sin embargo, esto no pudo ser confirmado en el caso de los hombres. Por tanto, se recomienda que futuros estudios indaguen estas diferencias y el efecto moderador del sexo, especialmente mediante muestras más amplias con mayor potencia estadística que permitan realizar generalizaciones.

Acerca del rasgo Extraversión, no se hallaron diferencias significativas con los valores normativos, por lo que parecería que el mismo no es definitorio de las diferencias 
entre judokas y población normotípica. Esto es congruente con lo indicado por otros autores que analizaron muestras de judokas o de deportes con características similares (e.g., Petar, 2011; Ruiz-Barquín, 2012).

Los resultados de este estudio deben ser interpretados a la luz de las limitaciones del mismo. En primer lugar, se trabajó particularmente con judokas de la categoría senior, excluyendo del estudio a las categorías previas. Si bien se optó por limitar la muestra a esta categoría por ser la que agrupa a los judokas que llevan más tiempo en la práctica de este deporte, el estudio de las categorías previas (cadete, junior, infantil) también reviste interés, especialmente en tanto que se ha mencionado la utilidad de deportes como el judo en el desarrollo de la motricidad y la percepción espacial (Guillen Pereira, Rojas Valladares, Formoso Mieres, Contreras Velázquez, y Estevez Pichs, 2019; Rodríguez Porras, Cabra Fonseca, y León León, 2018; UNESCO, 1997). En segundo lugar, la muestra comparativa de población general que se utilizó no fue recolectada de forma directa para este estudio. A pesar de que se trató de muestras extraídas de la misma ciudad, existen otros factores asociados al nivel educativo o el nivel socioeconómico, que no fueron considerados en este estudio y que podrían jugar un rol como moderadores de los hallazgos. En tercer lugar, otros aspectos psicológicos (como el bienestar psicológico, por ejemplo) no considerados en esta investigación merecen igual interés y relevancia, y deberían ser abordados en futuras investigaciones. Por último, la generalización de los hallazgos es limitada puesto que se trabajó solo con participantes argentinos, y el tamaño de la muestra fue reducido. Particularmente destacable es la limitación de la gran proporción de hombres respecto de la cantidad de mujeres, $y$, si bien esta distribución es normal en la disciplina (e.g., Ruiz-Barquín, 2005), especialmente en la categoría senior, fu- turos estudios deberían explorar la posibilidad de replicar estos resultados con un mayor número de participantes judokas mujeres.

A pesar de las limitaciones, el estudio supone un aporte al análisis del perfil de practicante de la disciplina del judo, lo cual ha sido solo escasamente explorado hasta ahora. También implica un aporte al estudio de las características de personalidad de los deportistas de combate en general. Los hallazgos aquí reportados sugieren la importancia de la compresión de la personalidad para el trabajo de la disciplina deportiva del judo. Además, los hallazgos respaldan lo indicado en el rastreo bibliográfico acerca de que estos rasgos de personalidad, también serían significativos en el rendimiento de los competidores. Por lo tanto, los profesores de judo podrían considerar la indagación acerca de la personalidad de sus estudiantes para implementar esos conocimientos en su práctica diaria y optimizar el rendimiento (González-Campos, Valdivia-Moral, Zagalaz, y Romero 2015; Olmedilla et al., 2018). El análisis de los aspectos psicológicos de la práctica deportiva, la comprensión clara de las diferencias interindividuales entre los atletas y el conocimiento de las habilidades y aptitudes necesarias en las distintas disciplinas, resulta fundamental para el desarrollo de intervenciones en distintos áreas y contextos deportivos que permitan a cada deportista alcanzar su máximo potencial (Laborde, Breuer-Weißborn, y Dosseville, 2013; Llames et al., 2017).

\section{Referencias bibliográficas}

Alexandru, M. A., y Răşcanu, R. (2013). Personality determinants at children and juniors in the game of tennis. Procedia-Social and Behavioral Sciences, 76, 536-541. https://doi.org/10.1016/j.sbspro.2013.04.160 
Allen, M. S., Greenlees, I., y Jones, M. (2013). Personality in sport: a comprehensive review. International Review of Sport and Exercise Psychology, 6(1), 184-208. https://doi.or/10.1080/175098 $\underline{4 x .2013 .769614}$

American Psychological Association (2010). Ethical principles of psychologists and code of conduct. Autor.

Bara Filho, M. G., Scipião Ribeiro, L. C., y Guillen García, F. (2004). La personalidad de deportistas brasileños de alto nivel: comparación entre diferentes modalidades deportivas. Cuadernos de Psicología del Deporte, 4, 117-127.

Barra Almagiá, E., Soto Quevedo, O., y Schmidt Díaz, K. (2019). Personalidad y bienestar psicológico: un estudio en universitarios chilenos. Revista de Psicología, 9(17), 7-18.

Bermúdez, J. (1995). Cuestionario "Big Five" (Adaptación al castellano del cuestionario BFQ de G.V. Caprara, Barbaranelli y Borgogni, 1993). Madrid: TEA Ediciones.

Carazo Vargas, P., y Araya Vargas, G. A. (2010). Perfil de rasgos psicológicos para el rendimiento deportivo en hombres y mujeres practicantes de taekwondo. $R e-$ vista Iberoamericana de Psicología del Ejercicio y el Deporte, 5(2), 253-265.

Catalá, P., y Peñacoba, C. (2020). Factores psicológicos asociados a la vulnerabilidad de lesiones. Estudio de caso en un equipo de fútbol semi-profesional. $R e$ vista de Psicología Aplicada al Deporte y al Ejercicio Físico, 5(1), e3. https://doi. org/10.5093/rpadef2020a5

Cavallera, G. M., Passerini, A., y Pepe, A. (2013). Personality traits and the role of gender in swimmers at the leisure level. Social Behavior and Personality: An International Journal, 41(4), 693-703. https:// doi.org/10.2224/sbp.2013.41.4.693
Chopik, W. J., y Kitayama, S. (2018). Personality change across the life span: Insights from a cross-cultural, longitudinal study. Journal of personality, 86(3), 508521. https://doi.org/10.1111/jopy.12332

Church, A. T. (2016). Personality traits across cultures. Current Opinion in Psychology, 8, 22-30. https://doi.org/10.1016/j. copsyc. 2015.09 .014

Cohen, J. (1988). Statistical power analysis for the behavioral sciences (2nd ed.). Mahwah: Erlbaum.

Corral Gregorio, S., Pamos de la Hoz, A., Pereña Brand, J., y Seisdedos Cubero, N. (2009). Test de Personalidad de TEA. Manual (4ta Ed.). Madrid: TEA Ediciones.

Costa, Jr., P. T., McCrae, R. R., y Löckenhoff, C. E. (2018). Personality across the life span. Annual Review of Psychology, 70(1), 423-448. https://doi.org/10.1146/annurev-psych-010418-103244

Costa, P. T., Terracciano, A., y McCrae, R. R. (2001). Gender differences in personality traits across cultures: Robust and surprising findings. Journal of Personality and Social Psychology, 81(2), 322-331. https:// doi.org/10.1037/0022-3514.81.2.322

Costa, P. T., y McCrae, R. R. (1985). The NEO Personality Inventory Manual. Lutz, FL: PAR.

De Miguel Negredo, A. (2005). Diferencias de edad y género en el NEOPIR en dos muestras con distinto nivel académico. International Journal of Psychology and Psychological Therapy, 5(1), 13-31.

De Vries, R. E. (2020). The main dimensions of sport personality traits: A lexical approach. Frontiers in Psychology, 11, 2211. https://doi.org/10.3389/ fpsyg. 2020.02211 
Field, A. (2009). Discovering statistics using SPSS. Thousand Oaks, CA: SAGE.

Garcés de Los Fayos Ruiz, E. J., Pérez Hernández-Ardieta, I., López María Dolores, J. C. (2002). Personalidad, diferencias individuales y ejecución deportiva. En A. Olmedilla Zafra, E. J. Garcés de Los Fayos Ruiz, y G. Nieto García (Coords.), Manual de Psicología del deporte (pp. 10523). Murcia: Diego Marín Librero Editor.

García-Naveira, A. (2010). Diferencias individuales en estilos de personalidad y rendimiento en deportistas [Tesis Doctoral]. Universidad Complutense de Madrid, España.

García-Naveira, A., Locatelli Dalimier, L., Ruiz-Barquín, R., y González, J. (2016). Diferencias en personalidad entre deportista de deportes de riesgo vs otras modalidades deportivas. Cuadernos de Psicología del Deporte, 16(3), 33-44.

García-Naveira, A., Ruiz-Barquín, R., y Pujals, C. (2011). Diferencias en personalidad en función de la práctica o no deportiva, nivel de competición y categoría por edad en jugadores de fútbol desde el modelo de Costa y McCrae. Revista de Psicología del Deporte, 20(1), 29-44.

García-Naveira, A., y Díaz-Morales, J. F. (2010). Relación entre optimismo/pesimismo disposicional, rendimiento y edad en jugadores de fútbol de competición. Revista Iberoamericana de Psicología del Ejercicio y el Deporte, 5(1), 45-60.

García-Naveira, A., y Ruiz-Barquín, R. (2013). La personalidad del deportista: una revisión teórica desde la perspectiva de rasgos. Revista Internacional de Medicina y Ciencias de la Actividad Física y del Deporte/International Journal of Medicine and Science of Physical Activity and Sport, 13(51), 627-645.
García-Naveira, A., y Ruiz-Barquín, R. (2016). Diferencias en personalidad en función de la práctica o no deportiva y categoría por edad en jugadores de fútbol de rendimiento desde el modelo de Costa y McCrae. Revista Iberoamericana de Psicología del Ejercicio y el Deporte, 11(1), 23-29.

Gee, C. J., Marshall, J. C., y King, J. F. (2010). Should coaches use personality assessments in the talent identification process? A 15 year predictive study on professional hockey players. International Journal of Coaching Science, 4(1), 25-34.

George, D., y Mallery, P. (2016). IBM SPSS statistics 23 step by step: A simple guide and reference (14th Ed.). Londres: Routledge.

González Hernández, J. G., Garcés de los Fayos Ruiz, E., y Ortega Toro, E. (2014). Avanzando en el camino de la diferenciación psicológica del deportista. Ejemplos de diferencias en sexo y modalidad deportiva. Anuario de Psicología/The UB Journal of Psychology, 44(1), 31-44. https://doi. org/10.1344/anuario.any.volum.numero

González Hernández, J., y Valadez Jiménez, A. (2016). Personalidad y respuesta psicológica en deportistas. Representación temporal y adaptativa del proceso persona-deporte. Retos: Nuevas Tendencias en Educación Física, Deporte y Recreación, 30, 211-215. https://doi. org/10.47197/retos.v0i30.50267

González, J., Garcés de los Fayos, E. J., López-Mora, C., y Zapata, J. (2016). Personalidad y estilos de toma de decisiones en la práctica deportiva. Revista Iberoamericana de Psicología del Ejercicio y el Deporte, 11(1), 107-112.

González-Campos, G., Valdivia-Moral, P., Zagalaz, M. L., y Romero, S. (2015). La autoconfianza y el control del estrés en 
futbolistas: revisión de estudios. Revista Iberoamericana de Psicología del Ejercicio y el Deporte, 10(1), 95-101.

Guillen Pereira, L., Rojas Valladares, L., Formoso Mieres, A. A., Contreras Velázquez, L. M., y Estevez Pichs, M. A. (2019). Influencia de la estimulación temprana en el desarrollo sensorio-motriz de niños de cuatro a seis años: una visión desde el Karate Do. Retos: Nuevas Tendencias en Educación Física, Deporte y Recreación, 35, 147-155. http://doi.org/10.47197/retos. v0i35.63104

Hernández Sampieri, R., Fernández Collado, C., y Baptista Lucio, P. (2014). Metodología de la Investigación (6ta ed.). México, DF: McGraw Hill.

Hughes, S., Case, H.S., Stuempfle, K. y Evans, D. (2003). Personality profiles of Iditasport Ultra-Marathon participants. Journal of Applied Sport Psychology, 15(3), 256-261. https://doi. org/10.1080/10413200305385

Ilyasi, G., y Salehian, M.H. (2011). Comparison of personality traits between individual and team athletes. Middle-East Journal of Scientific Research, 9(4), 527-530.

Jones, S. E., Miller, J. D., y Lynam, D. R. (2011). Personality, antisocial behavior, and aggression: A meta-analytic review. Journal of Criminal Justice, 39(4), 329-337. https://doi.org/10.1016/j.jcrimjus.2011.03.004

Kokko, K., Tolvanen, A., y Pulkkinen, L. (2013). Associations between personality traits and psychological well-being across time in middle adulthood. Journal of Research in Personality, 47(6), 748-756. https://doi.org/10.1016/j.jrp.2013.07.002

Laborde, S., Breuer-Weißborn, J., y Dosseville, F. (2013). Personality-trait-like individual differences in athletes. En C. Mohi- yeddini (Ed.), Advances in the Psychology of Sports and Exercise (pp. 25-60). Hauppauge: Nova Publishers.

Langvik, E., Hjemdal, O., y Nordahl, H. M. (2016). Personality traits, gender differences and symptoms of anhedonia: What does the Hospital Anxiety and Depression Scale (HADS) measure in nonclinical settings?. Scandinavian Journal of Psychology, 57(2), 144-151. https://doi.org/10.1111/ sjop.12272

Ledesma, R., Sánchez, R., y Díaz-Lázaro, C. (2011). Adjective Checklist to assess the big five personality factors in the Argentine population. Journal of Personality Assessment, 93(1), 46-55. https://doi.org/ $\underline{10.1080 / 00223891.2010 .513708}$

Llames, R., Escudero, J. T., Marí, P., Vives, J., y Martí, J. (2017). Investigadores académicos y profesionales de la Psicología del Deporte: Condenados a entenderse. Revista de Psicología del Deporte, 26(3), 80-84.

Lucas, R. E., y Donnellan, M. B. (2011). Personality development across the life span: Longitudinal analyses with a national sample from Germany. Journal of Personality and Social Psychology, 101(4), 847861. https://doi.org/10.1037/a0024298

Malinauskas, R., Dumciene, A., Mamkus, G., y Venckunas, T. (2014). Personality traits and exercise capacity in male athletes and non-athletes. Perceptual and Motor Skills, 118(1), 145-161. https://doi. org/10.2466/29.25.PMS.118k13w1

Manga, D., Ramos, F., y Morán, C. (2004). The Spanish norms of the NEO Five-Factor Inventory: New data and analyses for its improvement. International Journal of Psychology and Psychological Therapy, 4(3), 639-648.

Marín, A. A., Piñar, M., Camacho, P., Brox, V., Miranda-León, M., Suárez-Cadenas, 
E., ,.., y Cárdenas, D. (2012). Características de la personalidad de jóvenes jugadores de baloncesto (15-under). Cuadernos de Psicología del Deporte, 12(1), 135-138.

Matsumoto, D., Takeuchi, M., Nakajima, T., y lida, E. (2000). Competition anxiety, self-confidence, personality and competition performance of American elite and non-elite judo athletes. Research Journal of Budo, 32(3), 12-21.

McCrae, R. R. (2017). The Five-Factor Model across cultures. En A. T. Church (Ed.), The Praeger handbook of personality across cultures: Trait psychology across cultures (p. 47-71). Santa Barbara, CA: Praeger/ABC-CLIO.

McCrae, R. R., Costa, P. T., Ostendorf, F., Angleitner, A., Hřebíčková, M., Avia, M. D., ... y Smith, P. B. (2000). Nature over nurture. Temperament, personality, and life span development. Journal of Personality and Social Psychology, 78(1), 173-186. https://doi.org/10.1037/0022-3514.78.1.173

McCrae, R. R., y Costa Jr, P. T. (2021). Understanding persons: From Stern's personalistics to Five-Factor Theory. Personality and Individual Differences, 169, 109816. https://doi.org/10.1016/j.paid.2020.109816

McCrae, R. R., y Costa, P. (1990). Personality in adulthood. A five-factor theory perspective. Nueva York: Guildford Press.

Mirzaei, A., Nikbakhsh, R., y Sharififar, F. (2013). The relationship between personality traits and sport performance. European Journal of Experimental Biology, 3(3), 439-442.

Mladenović, M., Lazarević, P., Trunić, N., Bogavac, D., y Živković, M. (2017). Differences in personality features and achievement motivation in child athletes and non-athletes. Facta Universitatis, Series: Physical Education and Sport, 415-425.
Mortatti, M., y Scapula, C. A. (2014): Escala de Personalidad Resistente para Corredores: Estudios preliminaries [Tesis de grado]. Universidad Nacional de Mar del Plata, Argentina.

Nia, M. E., y Besharat, M. A. (2010). Comparison of athletes' personality characteristics in individual and team sports. Procedia Social and Behavioral Sciences, 5, 808-812. https://doi.org/10.1016/j.sbspro.2010.07.189

O'Hern, S., Stephens, A. N., Young, K. L., y Koppel, S. (2020). Personality traits as predictors of cyclist behaviour. Accident Analysis \& Prevention, 145, 105704. https://doi.org/10.1016/j.aap.2020.105704

Obmiński, Z., Mroczkowska, H., y Tomaszewski, W. (2016) Relationships between personality traits, resting serum hormones and visuomotor ability in male judokas. Annals of Agricultural and Environmental Medicine, 23(1), 79-83. https:// doi.org/10.5604/12321966.1197305

Olmedilla, A., Sánchez-Aldeguer, M. F., Almansa, C. M., Gómez-Espejo, V., y Ortega, E. (2018). Entrenamiento psicológico y mejora de aspectos psicológicos relevantes para el rendimiento deportivo en jugadoras de fútbol. Revista de Psicología Aplicada al Deporte y al Ejercicio Físico, 3(1), e8. https://doi.org/10.5093/rpadef2018a2

Parmigiani, S., Dadomo, H., Bartolomucci, A., Brain, P. F., Carbucicchio, A., Costantino, C., ... y Volpi, R. (2009). Personality traits and endocrine response as possible asymmetry factors of agonistic outcome in karate athletes. Aggressive Behavior, 35(4), 324-333. https://doi.org/10.1002/ ab.20306

Petar, M. (2011). Emotional competence, styles of coping with stressful situations, anxiety and personality traits in judokas. Serbian Journal of Sports Sciences, 5(4), 163-169. 
Piedmont, R. L., Hill, D. C., y Blanco, S. (1999). Predicting athletic performance using the five-factormodel of personality. Personality and Individual Differences, 27(4), 769-777. https://doi. org/10.1016/S0191-8869(98)00280-3

Rhodes, R., y Smith, N. (2006). Personality correlates of physical activity: A review and meta-analysis. British Journal of Sports Medicine, 40(12), 958-965. https:// doi.org/10.1136/bjsm.2006.028860

Rodríguez Porras, Y., Cabra Fonseca, L., y León León, E. (2018). La práctica del judo para el desarrollo de las habilidades motrices de niños y niñas de 6 a 9 años en la escuela rural el hato [Tesis de Grado]. Universidad Pedagógica Nacional, Colombia.

Rodríguez, M. (2003). Diagnóstico de personalidad en deporte de competición: fútbol [Tesis Doctoral]. Universidad Las Palmas de Gran Canaria, España.

Ruiz-Barquín, R. (2005). Análisis de las diferencias de personalidad en el deporte del judo a nivel competitivo en función de la variable sexo y categoría de edad deportiva. Cuadernos de Psicología del Deporte, 5, 29-48.

Ruiz-Barquín, R. (2006). Predicción del resultado deportivo en judokas cadetes de competición aplicando las dimensiones del cuestionario de personalidad BFQ. Revista Iberoamericana de Psicología del Ejercicio y el Deporte, 1(2), 69-88.

Ruiz-Barquín, R. (2008). Aportaciones del análisis subdimensional del cuestionario de personalidad BFQ para la predicción del rendimiento en judokas jóvenes de competición. Cuadernos de Psicología del Deporte, 8(1), 5-30.

Ruiz-Barquín, R. (2012). Relaciones entre características de personalidad y estados de ánimo: un estudio con deportistas de combate universitarios. Revista Iberoamericana de Psicología del Ejercicio y el Deporte, 7(1), 89-112.

Ruiz-Barquín, R., y García-Naveira, A. (2013). Personalidad, edad y rendimiento deportivo en jugadores de fútbol desde el modelo de Costa y McCrae. Anales de Psicología / Annals of Psychology, 29(3), 642-655. https://doi.org/10.6018/analesps.29.3.175771

Sánchez, R. O. (2019). Correr: físico más mente. Factores psicológicos relacionados al correr. Mar del Plata: Editorial de la Universidad Nacional de Mar del Plata.

Sánchez, R. O., y Ledesma, R. (2007). Los Cinco Grandes Factores: Cómo entender la personalidad y cómo evaluarla. En A. Monjeau (Ed.), Conocimiento para la transformación (pp. 131-160). Mar del Plata: Universidad Atlántida Argentina.

Sánchez, R. O., y Ledesma, R. D. (2013). Listado de Adjetivos para Evaluar Personalidad: Propiedades y normas para una población argentina. Revista Argentina de Clínica Psicológica, 22(2), 147-160.

Schmitt, D. P., Long, A. E., McPhearson, A., O'Brien, K., Remmert, B., y Shah, S. H. (2017). Personality and gender differences in global perspective. International Journal of Psychology, 52, 45-56. https:// doi.org/10.1002/ijop.12265

Schmitt, D., Realo, A., Voracek, M., y Allik, J. (2008). Why can't a man be more like a woman? Sex differences in Big Five personality traits across 55 cultures. Journal of Personality and Social Psychology, 94(1), 168-182. https://doi.org/10.1037/00223514.94.1.168

Schwaba, T., y Bleidorn, W. (2018). Individual differences in personality change across the adult life span. Journal of Personality, 86(3), 450-464. https://doi. org/10.1111/jopy. 12327 
Steca, P., Baretta, D., Greco, A., D'Addario, M., y Monzani, D. (2018). Associations between personality, sports participation and athletic success. A comparison of Big Five in sporting and non-sporting adults. Personality and Individual Differences, 121, 176-183. https://doi.org/10.1016/j.paid.2017.09.040

Trninić, V., Barančić, M., y Nazor, M. (2008). The five-factor model of personality and aggressiveness in prisoners and athletes. Kinesiology, 40(2), 170-181.

Trninić, V., Trninić, M., y Penezić, Z. (2016). Personality differences between the players regarding the type of sport and age. Acta Kinesiologica, 10(2), 69-74.

Trochim, W. M., y Donnelly, J. P. (2006). The research methods knowledge base (3rd ed.). Ohio: Atomic Dog.

UNESCO (1997). Plan de educación para el desarrollo y la integración de América Latina. Recuperado de: https://unesdoc. unesco.org/ark:/48223/pf0000146551 spa

Vaughan, R. S., y Edwards, E. J. (2020). Executive function and personality: The moderating role of athletic expertise. Personality and Individual Differences, 161, 109973. https://doi.org/10.1016/j. paid.2020.109973

Waleriańczyk, W., y Stolarski, M. (2021). Personality and sport performance: The role of perfectionism, Big Five traits, and anticipated performance in predicting the results of distance running competitions. Personality and Individual Differences, 169, 109993. https://doi.org/10.1016/j. paid.2020.109993
Weinberg, R. S., y Gould, D. (2010). Fundamentos de Psicología del deporte y del ejercicio físico. Buenos Aires: Editorial Médica Panamericana.

Weisberg, Y. J., DeYoung, C. G., y Hirsh, J. B. (2011). Gender differences in personality across the ten aspects of the Big Five. Frontiers in Personality Science and Individual Differences, 2, 178. https://doi. org/10.3389/fpsyg.2011.00178

Woodman, T., Zourbanos, N., Hardy, L., Beattie, S., y McQuillan, A. (2010). Do performance strategies moderate the relationship between personality and training behaviors? An exploratory study. Journal of Applied Sport Psychology, 22(2), 183-197. https://doi. org/10.1080/10413201003664673

World Medical Association (2013). Declaration of Helsinki - Ethical principles for medical research involving human subjects. Recuperado de: https://www.wma. net/policies-post/wma-declaration-of-heIsinki-ethical-principles-for-medical-research-involving-human-subjects/

Zhang, G., Chen, X., Xiao, L., Li, Y., Li, B., Yan, Z., ... y Rost, D. H. (2019). The relationship between big five and self-control in boxers: A mediating model. Frontiers in Psychology, 10, 1690. https://doi. org/10.3389/fpsyg.2019.01690 